University of Washington Tacoma

UW Tacoma Digital Commons

SIAS Faculty Publications

School of Interdisciplinary Arts and Sciences

$11-4-2013$

\title{
Diversity Perspectives and Minority Nonprofit Board Member Inclusion
}

Ruth Sessler Bernstein

University of Washington Tacoma, bernstrs@uw.edu

Diana Bilimoria

Follow this and additional works at: https://digitalcommons.tacoma.uw.edu/ias_pub

\section{Recommended Citation}

Bernstein, Ruth Sessler and Bilimoria, Diana, "Diversity Perspectives and Minority Nonprofit Board Member Inclusion" (2013). SIAS Faculty Publications. 645.

https://digitalcommons.tacoma.uw.edu/ias_pub/645

This Article is brought to you for free and open access by the School of Interdisciplinary Arts and Sciences at UW Tacoma Digital Commons. It has been accepted for inclusion in SIAS Faculty Publications by an authorized administrator of UW Tacoma Digital Commons. 


\title{
Diversity Perspectives and Minority Nonprofit Board Member Inclusion ${ }^{1}$
}

\author{
Ruth Bernstein \\ Mandel Center for Nonprofit Organizations \\ Weatherhead School of Management \\ Case Western Reserve University \\ Cleveland, Ohio \\ ruthbernst@aol.com \\ and \\ Diana Bilimoria * \\ Professor of Organizational Behavior \\ Weatherhead School of Management \\ Case Western Reserve University \\ Cleveland, Ohio \\ diana.bilimoria@case.edu
}

\section{Diversity Perspectives and Minority Nonprofit Board Member Inclusion}

\footnotetext{
${ }^{1}$ We thank Paul Salipante who helped in the framing of this study and provided useful comments throughout the writing process. We thank Robin Ely, Nicole Tysvaer, Judith Weisinger, and the two anonymous reviewers for their excellent suggestions to improve the manuscript. Our thanks also to BoardSource for providing us with their Vital Voices survey data and James Gaskin for aiding us in the statistical analyses.
} 


\begin{abstract}
Purpose: Using survey data of nonprofit board members from racial/ethnic minority groups we investigate how the three work group perspectives towards diversity theorized by Ely and Thomas (2001) - discrimination-and-fairness (P1), access-and-legitimacy (P2), and integrationand-learning (P3) - are associated with minority group members' inclusion experiences.

Design: We investigate how an organization's motivations for board diversity, as perceived by racial/ethnic minority board members, drive various organizational and board-level practices and behaviors, and ultimately impact their experience of inclusion. We use two different operationalizations of the diversity perspectives to assess their impact on minority board members' inclusion experiences. The hypothesized model was tested using partial least squares analyses on the responses of 403 racial/ethnic minority nonprofit board members.

Findings: Regardless of the measure used, racial/ethnic minority board members experienced increased feelings of inclusion as the perceived operating perspective for board diversity changed from P1 to P2 to P3, while concurrently the mediating factors influencing inclusion experiences changed in significance. Findings support the importance of the integration-and-learning perspective for the experience of inclusion by racial/ethnic minority board members.

Practical Implications: Findings indicate that organizations that employ an integration-andlearning approach to diversity and focus on encouraging their majority group members to engage in inclusive behaviors, rather than on policies and procedures, will engender the racial/ethnic minorities' experience of inclusion.

Originality: This study quantitatively investigated how three organizational diversity paradigms are associated with the individual inclusion experiences of minority nonprofit board members.
\end{abstract}

Key words: Diversification; Ethnic minorities; Management culture; Organizational culture 
The full inclusion of racial/ethnic minority group members on boards of trustees remains an elusive goal for many nonprofit organizations. In this study, we examine the key factors that contribute to the inclusion experience of racial/ethnic minority members serving on nonprofit boards. In particular, we investigate how an organization's motivations for board diversity, as perceived by racial/ethnic minority board members, drive various organizational and board-level practices and behaviors, and ultimately impact their experience of inclusion. These findings will aid organizations in understanding how their perspectives and approaches toward diversity may hinder or facilitate their success in achieving the full benefits of workforce diversity.

The impetus for this research are the findings that only $28 \%$ of chief executives and board members surveyed were satisfied with the racial/ethnic diversity of their boards (Walker \& Davidson, 2010) and that persons of color (non-Caucasians) made only extremely slight gains (from $14 \%$ to $16 \%$ ) in their overall participation on the boards of nonprofit organizations between 1993 and 2010 (BoardSource Governance, in review). The BoardSource Nonprofit Governance Index 2010 found that of all nonprofits surveyed one-third did not have any minority representation on their boards and $48 \%$ had boards with one-quarter or fewer members representing racial and/or ethnic minority backgrounds. The Index reported that among racial/ethnic minority nonprofit board members $46 \%$ felt that the board on which they served was, to a great or very great extent, interested in becoming more diverse in order to expand the diversity of thought and $57 \%$ reported that their board, to a great or very great extent, desired to be more diverse in order to increase their ability to serve the mission. These statistics indicate the importance of studying boardroom diversity in nonprofit organizations, and the need for organizations to understand how they can better include diverse members to achieve desired outcomes. 
Drawing on extant conceptualizations in the literature (e.g., Pelled, Ledford \& Mohrman: 1999; Mor Barak, 2000; Roberson, 2006; Janssens and Zanoni, 2007), we define inclusion as an individual's or subgroup's sense of efficacy, belonging, and value in a work system. Ely and Thomas (2001) identified three perspectives on work group diversity that have been effective in motivating managers to diversify their staffs and engender inclusion: discrimination-and-fairness (P1), access-and-legitimacy (P2), and integration-and-learning (P3). These authors theorize that only the third, integration-and-learning, perspective will result in full inclusion of minority perspectives in organizational structures, work groups and relations, and ultimately achieve sustained benefits from diversity; the other two perspectives may result in short run gains in minority representation but workplace benefits will fail to be fully realized and the gains will be difficult to sustain. While this proposition is theoretically attractive and intuitive, to our knowledge no empirical test of the influence of the three diversity perspectives on the inclusion experience of individuals has been undertaken. We investigate the association of the three diversity perspectives with various board-level and organizational-level diversity practices and behaviors as well as their ultimate influence on the inclusion experience of minority board members. Knowledge gained by this research will enable organizational leaders in nonprofit and other organizations to more successfully create cultures of diversity in which members of all groups feel fully included.

The rest of this paper is structured as follows. First, we examine relevant literature and key concepts to develop a theoretical framework and hypotheses to guide the analyses. We then describe the research design and methods, and discuss the main findings. Subsequently, we discuss the study's contributions and limitations as well as the implications of the findings for future research and organizational practice. 


\section{THEORY DEVELOPMENT AND HYPOTHESES}

Figure 1 illustrates the hypothesized relationships among the three diversity perspectives, board and organization level diversity behaviors and practices, and a minority board member's individual experience of inclusion. The inclusion experienced by minority board members is important because it has consequences for their recruitment, performance and retention, all indicators of successful diversification at the board level.

Figure 1 about here

\section{Diversity Perspectives}

Ely and Thomas (2001) noted that of particular importance regarding diversity is what people believe. The value of diversity maintains that diverse groups, as opposed to homogeneous groups, provide a broader range of information, knowledge, and perspectives (Cox, Lobel, \& McLeod, 1991). Ely and Thomas proposed their three perspectives for fostering and managing work group diversity in order to seek its benefits. Ely and Thomas examined how feeling valued and respected mediated the impact of diversity in cultural identities on work group functioning. This paper differs in placing individual inclusion experiences as the dependent variable. The discrimination-and-fairness perspective (P1) strives to establish "a culturally diverse workforce as a moral imperative to ensure justice and fair treatment of all members of society... with no instrumental link between diversity and group's work" (Ely \& Thomas, 2001, pp. 245-246). Underlying this approach to diversity is the motivation to do the right thing for all people by creating an equal opportunity and equal treatment workplace. P1 promotes assimilation based on sameness, promoting valuing and treating all employees equally. However, this perspective ignores the potential for employees' culturally based differences to benefit the organization and 
often creates cross-cultural tension, racial discrimination problems, with women and people of color reporting lack of respect and value.

The access-and-legitimacy perspective $(P 2)$ is predicated on the acceptance and celebration of differences. This perspective strives for representative diversity - diversity that mirrors the diversity in the community. This approach allows work groups to operate in business environments by hiring employees of similar demographic characteristics as customers in order to gain access to new markets and foster better relationships with minority customers. This focus on differentiation sometimes causes employees to feel devalued due to their culture or ethnicity. P2 is problematic because people's identity-related perspectives are not brought to bear on the core work of the organization, only in those places deemed relevant, which tends to be at the organization's boundaries, where they are interacting with its market or constituencies. This perspective often leaves culturally dissimilar individuals feeling appreciated only for a specific characteristic (e.g., race/ethnicity) not for their potential contributions in a range of functions.

The integration-and-learning perspective $(P 3)$ focuses on valuing diverse members of a workforce as contributing different beneficial perspectives and approaches from which all employees learn. It draws on the two previous paradigms to promote equal opportunity and acknowledge and recognize cultural differences in order that "cultural identity shapes how people experience, see, and know the world" enabling cultural differences to be a "source of insight and skill” (Ely \& Thomas, 2001, p. 241). P3 encourages all organizational members to accept differences and learn from them new ways to improve organizational performance. When work groups foster environments that promote learning, diversity may be appreciated as a resource, not an impediment, to the organization, potentially, enhancing performance (Ely, Padavic, \& Thomas, 2012). 
We hypothesize that each perspective proposed by Thomas and Ely likely has a different impact on the individual inclusion experienced by minority board members, as follows: The lack of a diversity perspective (e.g., a board that does not take diversity concerns into account at all) would likely lead to negative inclusion experiences. The perceived discrimination-and-fairness (P1) perspective is likely to yield the lowest experience of inclusion by minority board members since this diversity perspective emphasizes organizational compliance and assimilation of differences. The perceived integration-and-learning (P3) perspective is likely to yield the highest experience of inclusion because diverse individuals are valued for their talents and potential, and learning from differences is encouraged. The perceived access-and-legitimacy P2 perspective is likely to yield an experience of inclusion somewhere between the other two perspectives since under this perspective individual differences are acknowledged but are instrumentally valued for their contributions. We hypothesize that each subsequent diversity perspective is an improvement over the previous one, and hence we propose a progression from weak to moderate to strong from $\mathrm{P} 1$ to $\mathrm{P} 2$ to $\mathrm{P} 3$ perspectives. Thus,

Hypothesis 1a. There will be a weak positive association between PI and IIE.

Hypothesis $1 b$. There will be a moderate positive association between P2 and IIE. Hypothesis 1c. There will be a strong positive association between P3 and IIE.

\section{Conditions Facilitating Inclusion - Board and Organizational Behaviors and Practices}

Ely and Thomas (2001) presented intermediate outcomes that mediate their theorized effects of the three diversity perspectives on group functioning, two of which are directly relevant for our study: quality of intergroup relations and feeling valued and respected. Our dependent variable, Individual Inclusion Experiences, reflects a minority board member's feeling valued and respected. Ely and Thomas' other relevant mediator, the quality of intergroup 
relations, captures how different groups are accorded power and status, and how majority groups treat minority groups. One of our mediating constructs, Board Inclusion Behaviors, directly reflects Ely and Thomas's quality of intergroup relations by including the concepts of equal treatment of all groups and inclusive behaviors and discussions. These behaviors at the board level impart to the entire organization expected behaviors towards others. Our two other mediating constructs between diversity perspectives and the experience of inclusion, Board Inclusion Practices and Organizational Inclusion Practices, are more focused on specific practices and policies adapted at the board and organizational levels to further workforce diversity.

Ely and Thomas (2001) noted that for diversity benefits to be significant and sustainable, a set of conditions is helpful. For example, organizational culture must create high performance standards that apply to everyone, foster high quality intergroup relations, be attentive to the meaning and significance of people's cultural identities, and make all employees feel valued and respected. The organization must have a clear mission and operate in a fair, egalitarian, and nonbureaucratic structure. Ely and Thomas propose that these preconditions enable work groups to actively explore employee differences while positively affecting employee relationships and how work is accomplished. The value of board diversity lies in increased innovation (e.g., Miller and Triana, 2009) and firm performance (e.g., Erhardt, Werbel, \& Sharder, 2003). Ely et al. (2012) and Kochan. Bezrukova, Ely, Jackson, Joshi, Levin, \& Thomas (2003) suggested that strong managerial commitments to diversity generally eliminate the potentially negative effects of diversity on workgroup processes and performance. These authors recommended that requiring a sustained, systemic approach and long-term commitment with an opportunity for group learning might leverage diversity benefits. Achieving these goals may necessitate taking a long-term 
view with a focus on learning and the development of tacit knowledge about intercultural interactions (Weisinger \& Salipante, 2007).

Operationalizing these ideas, we theorize that three facilitating conditions may derive from the diversity perspectives and be particularly relevant for board members' experience of inclusion - board inclusion behaviors, board inclusion practices, and organizational inclusion practices - as described below.

Board Inclusion Behaviors (BIB) describe the intragroup communication, influence and power interactions that the dominant members of small groups engage in consciously or unconsciously which signal the authentic inclusion of diversity. Examples of such behaviors may be whether communications and decision power are geared to, and maintained by, a dominant group and whether insensitive or offensive comments or jokes are encountered and tolerated. Such behaviors may be perceived and interpreted by minority members as reflecting their true value and treatment by majority members, influencing a minority board member's experience of inclusion. This construct is suggestive of the majority board members having intercultural competence and communication (Perry \& Southwell, 2011).

In addition to the likely direct association between BIB and the experience of inclusion of minority board members, it is likely that each of the three perceived diversity perspectives may result in different board inclusion behaviors, with the least inclusive board-level interactions deriving from the engagement of diversity from the P1, and the most inclusive deriving from the engagement of diversity from the P3, as follows.

Hypothesis 2 a. There will be a weak positive association between P1 and BIB. Hypothesis $2 b$. There will be a moderate positive association between P2 and BIB. Hypothesis 2c. There will be a strong positive association between P3 and BIB. 
Hypothesis $2 d$. BIB will be positively associated with IIE.

Board Inclusion Practices (BIP) describe practices and procedures that are commonly believed to enhance diversity and improve the experience for minority group members, such as diversity statements, policies, committees of taskforces dedicated to diversity and inclusion, diversity training for board members, and integration of diversity into the core mission and values. One third of respondents in a recent survey indicated that having such policies and practices was the second most important route to inclusivity (BoardSource, 2009). Bradshaw and Fredette (2012) suggest that adoption of diversity practices and policies at the board level have more success in recruiting minority board members. Management practices such as inclusion of explicit statements allow members to critically reflect on the organization's norms and values and eventually initiate changes in their cognitive frames and schemas (Hanappi-Egger, 2012). The effective use of board inclusion practices is thus likely to positively influence a minority board member's experience of inclusion.

In addition to the likely direct association between BIP and the experience of inclusion of minority board members, it is likely that each of the three perceived diversity perspectives may result in different board inclusion practices, with the least inclusive board practices deriving from the engagement of diversity from the first perspective, and the most inclusive board practices deriving from the engagement of diversity from the third perspective, as follows.

Hypothesis $3 a$. There will be a weak positive association between P1 and BIP. Hypothesis $3 b$. There will be a moderate positive association between $P 2$ and BIP. Hypothesis 3c. There will be a strong positive association between P3 and BIP. Hypothesis $3 d$. BIP will be positively associated with IIE.

Organizational Inclusion Practices (OIP) refer to organizational efforts to support 
diversity. Organizational inclusion practices describe organization-wide policies and practices related to diversity and inclusion such as perceptions of the extent to which the organization is committed to diversity and inclusion, engages in recruitment efforts that reach diverse communities, and engages in organizational communications that reflect the needs of diverse communities. Inclusion policies and practices, while frequently adopted by organizations, have not previously been shown to directly impact individual inclusion experiences.

The effective use of organizational-level inclusion practices is thus another likely way to influence a minority board member's experience of inclusion. As with the BIB and BIP, we hypothesize that there will be a direct positive association between OIP and minority board members' experience of inclusion. Additionally, it is likely that each of the three perceived diversity perspectives may result in different organizational inclusion practices, with the least inclusive organizational practices deriving from the engagement of diversity from the first perspective, and the most inclusive organizational practices deriving from the engagement of diversity from the third perspective, as follows.

Hypothesis 4a. There will be a weak positive association between P1 and OIP.

Hypothesis 4b. There will be a moderate positive association between P2 and OIP.

Hypothesis 4c. There will be a strong positive association between P3 and OIP.

Hypothesis 4d. OIP will be positively associated with IIE.

\section{METHODOLOGY}

\section{Sample}

The sample consisted of 403 nonprofit board members from racial/ethnic minority groups. The survey, entitled Vital Voices, was developed and conducted by BoardSource, a 
nonprofit organization dedicated to building exceptional nonprofit boards. Conducted in October 2009 the online survey was sent to board members belonging to previously identified racially and ethnically diverse groups in the wide range of BoardSource member nonprofit organizations. Subsequently, the survey link was forwarded, through networking, to additional board members of racial/minority groups. This study is a secondary analysis of the Vital Voices data. Fortuitously, the survey provided items that corresponded to important diversity constructs.

The respondents included in our analysis met two criteria: Individuals who (1) selfselected themselves as people of color, and (2) currently serve or formerly served on one or more "mainstream" nonprofit boards. Participants were instructed that mainstream in this context means organizations not organized around a particular racial or ethnic group. The sample has two limitations. First, each respondent filled out one survey only, but may have served on multiple boards. It is not possible to identify which board a respondent may have had in mind when s/he completed the survey. Second, multiple respondents may have served on the same board.

Demographic details of the respondents are provided in Table 1.

Table 1 about here

\section{Measures}

Individual Inclusion Experience (IIE) consisted of four items describing a minority board member's experience while serving on a board. Respondents were asked to indicate the extent to which they were comfortable voicing ideas, opinions, and discussing issues of diversity, felt valued and encouraged to be themselves by other board members, and felt they had the same opportunities as others for leadership and officer positions. Responses were recorded using a 
four-item Likert scale from never (1) to almost always (4). Cronbach's Alpha for IIE was 0.841.

Board Inclusion Behavior (BIB) included six items focused on majority group board members' behaviors. Respondents indicated the extent to which they had encountered insensitive or offensive comments or jokes, felt power was maintained by the dominant group, board communications were geared to the dominant group, inclusivity was discussed but not acted upon, and whether they felt as if they were treated differently than other board members because of their race/ethnicity. All six items were reverse-coded and employed a four-point scale from not-at-all (1) to very great extent (4). Cronbach's Alpha for BIB was 0.857.

Board Inclusion Practices (BIP) consisted of four items asking respondents to indicate the extent the following activities were effective in helping boards incorporate inclusive practices: (a) diversity statement, (b) a committee or task force dedicated to diversity and inclusion, (c) the inclusion of diversity as one of the organization's core values, and (d) diversity training. These items employed a four-point scale from not-at-all (1) to very great extent (4). Cronbach's Alpha for BIP was 0.844.

Organizational Inclusion Practices (OIP) consisted of seven items asking the extent to which the organization has policies and practices that address diversity and inclusion, is committed to diversity and inclusion, makes an effort to identify and address non-inclusive behaviors, has recruitment efforts that reach communities of color, assesses board culture and potential barriers to inclusion, and engages in communications that reflect the needs of communities of color. These items were recorded on a four-point scale from not-at-all (1) to very great extent (4). Cronbach's Alpha for OIP was 0.871.

Discrimination-and-Fairness Perspective (P1) was measured by one item. Using a fourpoint scale from not-at-all (1) to very great extent (4) respondents indicated the extent to which 
their board was motivated to become more diverse because "it was the right thing to do." While having only one item representing this perspective may be limiting, the item is very descriptive of the P1 perspective. Access-and-Legitimacy Perspective (P2) was measured by two items that emphasized serving the community and not the organization or individual. Using a four-point scale from not-at-all (1) to very great extent (4), respondents indicated the extent to which their board was motivated to become more diverse because diversity would "increase the board's ability to understand community needs" and "be a more effective way to represent the communities served”. Cronbach's Alpha for P2 was 0.787. Integration-and-Learning Perspective (P3) was measured by two items, using a four-point scale from not-at-all (1) to very great extent (4), indicating the extent to which respondents thought that their boards were motivated to become more diverse in order to "expand the diversity of thought" and "increase the board's ability to serve the mission”. Cronbach's Alpha for P3 was 0.699.

We controlled for additional variables that may be associated with variance in minority board members' experience of inclusion: (a) length of a board member's time on the board (less than one year $=1,1-2.9$ years $=2,3-6$ years $=3$, and more than 6 years $=4)(b)$ whether the respondent had ever served as board chair (Yes $=1$; No $=2)$, (c) board member ethnicity (American Indian or Alaska Native = 1; African American/Black; Asian = 2; Caucasian = 3; Hispanic, Latino, or Spanish $=4$; Native Hawaiian or Pacific Islander $=5$; Two or more races $=$ 6; Other =7), (d) board member gender $(\mathrm{M}=1 ; \mathrm{F}=2)$, and (e) size of the metropolitan area in which the board was located (Rural to semi-rural: $<50,000$; Small urban area: 50,000-249,000; Mid-size urban area: 250,000-499,000; Large urban area: 500,000-1 million; Mega urban area: > 1 million).

Since the above operationalization of $\mathrm{P} 1, \mathrm{P} 2$ and $\mathrm{P} 3$ is based on a conceptualization of the 
diversity perspectives as operating independently of each other, we employed a second operationalization of the perspectives by drawing on a different interpretation - that the perspectives are sequential and concentric. That is, the three perspectives also may be conceptualized in terms of an organization's employment of P2 as following and encompassing their adoption of P1, and employment of P3 as following and encompassing their adoption of P2 and P1 approaches. In other words, organizations that achieve an integration-and-learning perspective on diversity likely will also have adopted key elements of the access-and-legitimacy and the discrimination-and-fairness perspectives.

To operationalize this second interpretation, we calculated three new values for P1, P2 and P3 and called them $\boldsymbol{P 1 E}, \boldsymbol{P 2 E}$ and $\boldsymbol{P 3 E}$ (with the "E" reflecting the encompassment) based on the interpretation that as an organization progresses from $\mathrm{P} 1$ to $\mathrm{P} 3$, more advanced diversity perspectives encompass the effects of earlier perspectives. These new operationalizations were created by identifying the above-median responses to P1, P2 and P3 items as follows: P1E $(\mathrm{N}=61)$ was defined as the responses which were above the median on P1 items and below the median on P2 items and P3 items. P2E (N=53) was defined as the responses which were above the median on P2 items and P1 items. P3E (N=175) was defined as responses which were above the median on P3 items and either or both P1 or P2 items were above the median. Interestingly, 114 respondents did not identify their boards as above the median in any of the three perspectives, and these cases were excluded from the second operationalization analyses. Analyses of the P1E-P3E perspectives were conducted using the same model and statistical methods as used for P1-P3.

\section{Data Analysis}


The hypothesized model was tested using Partial Least Squares (PLS) (Chin \& Frye, 1998). PLS is not co-variance-based, allowing it to be used with data that does not meet normality assumptions (Chin, 1998). PLS was selected for use in the data analysis because the univariate analysis confirmed that this study did not meet the normality assumptions. The 403 responses selected had no missing values in the construct items and fewer than $3 \%$ missing cases in the control variables. These were imputed using the widely accepted "median of nearby points substitution" method (Hair et al., 2010). This assured the minimum threshold would be met based on an alpha level of $0.05,26$ predictors, an anticipated effect size of 0.15 , and a desired statistical power of 0.8 .

Exploratory factor analysis was conducted in SPSS using Principal Axis Factoring and Promax rotation. Some items were removed according to empirical evidence and theory, including loading values below 0.5, standard errors greater than 0.1, and T-values less than 1.96 (Chin, 1998). The final trimmed model presented 26 items yielding a 5-factor solution with items loading a piori. The total variance explained in by the model was $55.7 \%$.

\section{RESULTS}

Tables $2 \mathrm{~A}$ and 2B provide the descriptive statistics, correlations, factor loadings, composite reliability, and convergent and discriminant validity for P1-P3 and P1E-P3E. For all items in each construct, factor loadings were equal to or exceeded .60, composite reliability was above .70, and average variance extracted exceeded .50 (Chin, 1998).

Table 2A and Table 2B about here

The measurement model obtained using AMOS resulted in excellent fit statistics, Chi- 
squared $=534.337, \mathrm{df}=269, \mathrm{CMIN} / \mathrm{df}=1.986, \mathrm{CFI}=0.961, \mathrm{PCFI}=0.796, \mathrm{RMSEA}=0.044$

(0.039-0.050), and PCLOSE $=0.962$. The reliance on a single instrument for data collection necessitated examination for common method bias. We used four methods: (1) Harman single factor test (Podsakoff \& Organ, 1986), (2) examination of the correlation matrix (Tables 2A \& 2B) of the latent constructs for correlations above 0.90 (Pavlou, Laing, \& Hue, 2007), (3) addition of a common factor (adapted from Podsakoff, MacKenzie, Lee, \& Podsakoff, 2003), and (4) addition of a marker variable (Liang, Saraf, Hu, \& Xue, 2007). The results suggested that while common method variance is present, it is not strong enough to produce significant bias.

\section{Tests of Hypotheses}

Figures $2 \mathrm{~A}$ and $2 \mathrm{~B}$ and Table 3 provide the complete model results.

Figure 2A and Figure 2B about here

\section{Results of Operationalization 1 of P1, P2 and P3 Diversity Perspectives. Figure 2A}

indicates the results of the P1-P3 model (operationalization 1), revealing significant results for the three diversity perspective constructs. R-squared values were significant and sufficient to meet the acceptable power threshold (Hair et al., 2010). All of the paths emanating from discrimination-and-fairness (P1) were insignificant causing the P1 construct to be eliminated. In the model, the path from BIP to IIE was not significant. Additionally, the only significant path (p $<0.1)$ from $\mathrm{P} 2$ led to OIP. While P3 had a strong effect on BIP, this latter construct failed to impact IIE. All other path coefficients had substantive impact and were significant as tested by examination of the t-values generated through bootstrapping in PLS and by the Pseudo F test (Chin, 1998). Further, the findings of Figure 2A indicate that only P3 had strong significant effects on all three mediators and IIE. This demonstrates the strength of P3 both directly and 
indirectly in creating a sense of inclusion for minority board members. Overall, the results indicate that, as hypothesized, minority board members experienced increasingly inclusive experiences as the diversity perspectives progressed from P1 to P3. Of the controls—board chair, time on the board, gender, population size of the community, and respondent ethnicityonly gender exhibited a significant impact on IIE. The impact of gender on IIE was negative, indicating that women board members were less likely to experience inclusion.

Results of Operationalization 2 of Diversity Perspectives. Figure 2B illustrates the model using the diversity-encompassing perspectives P1E-P3E (operationalization 2). This analysis differed by having additional significant relationships, yet provided further evidence of minority board members experiencing increasingly inclusive experiences as the diversity perspectives progressed from P1E to P3E. R-squared values were significant and sufficient to meet the acceptable power threshold (Hair et al., 2010). P1E, P2E, and BIP had no significant direct impacts on IIE. BIB mediated the impact of P2E (fully) and P3E (partially). OIP partially mediated the impact of P3E on IIE. The impact of gender on IIE was negative, again indicating that women board members were less likely to experience inclusion. Figure 2B findings further confirm the effects of the integration-and-learning perspective. P3E strongly impacted the practices and behaviors of the organization and the board, as well as had a direct effect on IIE. BIB also resulted in strong positive IIE. The P3E results suggest that no matter what the respondents' perception of $\mathrm{P} 1$ and $\mathrm{P} 2, \mathrm{P} 3$ perceptions had a major impact on minority board members' individual inclusion experiences.

Table 3 provides a summary of the tests of hypotheses. As shown, significant differences exist between the three diversity perspectives. The impact of the diversity perspectives on minority Individual Inclusion Experiences (IEE) is significant only for the integration-and- 
learning perspective (P3), indicating that our first hypothesis, H1a, b, \& c, is supported. This hypothesis predicted that $\mathrm{P} 3$ would have a strong positive effect on IIE and that this effect would be stronger than that found between the access-and-legitimacy perspective (P2) and IIE and between the discrimination-and-fairness perspective (P1) and IIE. A similar relationship was revealed between the diversity perspectives and the three inclusion behaviors and practices, supporting $\mathrm{H} 2, \mathrm{H} 3$ \& $\mathrm{H} 4$. In other words, the strength of the effects between the diversity perspectives and Board Inclusion Behaviors (BIB), Board Inclusion Practices (BIP), and Organizational Inclusion Practices (OIP) is strongest for P3. In each of these cases $\mathrm{P} 1<\mathrm{P} 2<\mathrm{P} 3$. With P3, BIB becomes a significant construct and mediator between the diversity perspectives and IIE. In contrast, the influence of OIP on IIE is less influential and less significant with P3. These findings were replicated using P1E-P3E.

Table 3 about here

\section{DISCUSSION}

This study investigated how Ely and Thomas' three organizational diversity paradigms, discrimination-and-fairness (P1), access-and-legitimacy (P2), and integration-and-learning (P3) are associated with the individual inclusion experiences of minority board members who serve on nonprofit boards. The results indicated a direct path between the P3 diversity perspective and the inclusion experience of minority board members, demonstrating that individuals feel most included when they perceive they are valued for their talents, contributions and abilities to assist the board serve its mission and feel less included when they perceive $\mathrm{P} 3$ perspective to be lacking. The lack of significant direct relationships between the P1 or P2 diversity perspectives and inclusion suggest that individuals may be indifferent to these organizational perspectives 
more than they are associated with a lack of inclusion. These findings thus provide evidence to support the main thesis forwarded by Ely and Thomas.

The findings indicated that the organizational condition of BIB was strongly associated with minority board members' experience of inclusion. That is, when boardroom behavior focused on respect for individuals, treated all board members as equals, opened leadership positions to everyone, and did not tolerate individuals being less than decent to one another, minority board members experienced inclusion.

The findings also show that BIP were not significantly associated with IIE. Recall that BIP consisted of practices and procedures commonly believed to enhance diversity and improve the experience for minority group members, such as diversity statements, policies, committees of taskforces dedicated to diversity and inclusion, diversity training for board members, and integration of diversity into the core mission and values. Previous empirical evidence has shown that diversity-training programs often fail to achieve their goals and may even contribute to increased conflict and tension (Hemphill \& Haines, 1997) due to an overemphasis on individual differences which often results in defensiveness and perpetuation of stereotypes (Cox, 2001). Ely (2004) determined that diversity education programs did not "foster a positive relationship between diversity and performance" (p. 776), if anything, they created a social trap problem lowering achievement on some outcomes. Expecting diverse others, when placed together in groups, to develop relationships and learn from one another is unlikely. The circumstances of the interactions must be "favorable" (Amir, 1969) and include processes that will change attitudes (Pettigrew, 1998).

The insights gained from the present study may assist organizational leaders as they strive to create cultures of diversity in which members of all groups may feel included. The 
findings that BIB and the $\mathrm{P} 3$ perspective significantly impact IIE suggest the importance of creating an inclusive culture. Chavez and Weisinger (2008) emphasize the need for the creation of an inclusive "culture of diversity" with a long-term relational approach that emphasizes an attitudinal transformation that allows for learning and for barriers to be broken, while capitalizing on the distinctive perspectives of diverse individuals.

Our findings suggest that when the board adopts a culture of diversity that reflects a P3 perspective, minority board members feel equal and valued, instead of just being token minority members who allow majority board members to feel that they have diversified the board. Consistent with the integration-and-learning perspective (P3), Shore, Randel, Chung, Dean, Dean, Ehrart, \& Singh's (2011: 1265) review of inclusion and diversity in work groups suggests diverse work groups "incorporate both uniqueness (through viewing diversity as a resource) and belongingness (through members feeling valued and respected)". Conversely, if the members of racial/ethnic minority groups have low uniqueness and high belongingness they fit into the discrimination-and-fairness perspective (P1); and those with low belongingness and high value of uniqueness are reflected in the access-and-legitimacy perspective (P2). Shore et al. offer no single practice that will foster inclusion, and instead suggest that organizations enable all members to achieve insider status, adopt resolution procedures, improve communication facilitation, foster information sharing, enhance participation in decision making, provide freedom from biases and stereotypes, and be given a voice.

\section{Study Limitations}

The Vital Voices survey data had some limitations that impacted our study. We were unable to ascertain the precise survey response rate. The survey provided no information regarding the size of the board and the percentage of racial/ethnic minority group members 
serving on each board, and hence these potentially important variables could not be analyzed. We were further limited by having only one item to assess P1, the discrimination-and-fairness perspective. Other items were unusable due to lack of convergent reliability. We are confident that the one item-the board increased its diversity only in order to "do the right thing"sufficiently represented the P1 perspective. This study focused only on current nonprofit board members. Future research could collect data from racial/ethnic minority group members who are no longer serving on the boards because they never achieved a sense of inclusion, exploring whether these boards behaved in a manners inconsistent with the P3 perspective. Despite these limitations, this study provides a first rigorous quantitative examination of Ely and Thomas' theory, producing findings with important implications for future research and nonprofit leader's actions.

\section{Implications for Future Research}

While the control variables were selected in order to focus on just the conditions facilitating inclusion, it would be worthwhile to consider the impact on inclusion by individuals from different racial/ethnic minority groups who have spent more time on the board. Individuals from racial/ethnic minority groups who have served as president of their board, may perceive this as an ultimate vote of acceptance, increasing their feeling of inclusion within the group. This suggests that further study into the impact of tokenism on inclusion (i.e., when boards are only minimally diverse) is warranted.

The integration-and-learning paradigm (P3) points to work group success in situations where individuals can feel valued and a balance of belongingness and uniqueness (Shore et al., 2011). Future research that explores and identifies the nature and components of such inclusive cultures - their antecedents, dynamics and consequences - would inform organizational leaders 
and human resource specialists. We also urge further exploration of the organizational conditions facilitating inclusive cultures and inclusive leadership. In particular, Board Inclusion Behaviors, which had a very strong mediating impact in our study, is composed of items reflecting the types of behaviors we expect to see in an inclusive organization, including acting in an inclusive manner, being polite and respectful, and treating all board members equally. Organizational Inclusion Practices, suggestive of an organization willing to embrace diversity, while less impactful, are likely necessary for recruiting racial/ethnic minority members to the board. Future studies should replicate and further analyze the facilitating and constraining conditions of inclusion.

Consistent with Ely and Thomas's theory, an alternative approach would be to reframe the antecedents and consequences of the diversity perspectives. This would enable examining whether the diversity perspectives, $\mathrm{P} 1, \mathrm{P} 2$, and P3, mediate the impact of the behaviors and practices of the board/organization on the individual board member's inclusion experience. In addition, future research could delve into the power relation shift toward racial and ethnic minority members that Ely and Thomas project takes place with the adoption of the P3 perspective.

\section{Implications for Practice}

Our findings suggest that boards and organizations that focus on encouraging their majority group members to engage in inclusive behaviors, rather than focus only on policies and procedures, will engender more of the experience of inclusion by racial/ethnic minorities. While we found no direct effect between BIP and IIE, it is possible that diversity policies, procedures and training still serve as an entry point to increase awareness of these issues. It may be that high quality policies, procedures and trainings are a necessary, but not sufficient characteristic of 
inclusive environments. Focusing on majority board members' behaviors, including the desire to act upon being inclusive, not tolerating insensitive or offensive comments, ensuring that the dominant group does not exclusively control power, gearing board communications equally toward all members, and creating an environment where racial/ethnic minority group members are treated equally, would contribute to the creation of a positive organizational culture of inclusion. This study, and a number of preceding ones discussed here, point to success factors for diversity that seem to be outside of the realm of current diversity practices that emphasize compliance with policies and procedures. Our findings present an opportunity for nonprofit and diversity research to guide organizational leaders away from such potentially less effective practices and toward more effective actions of everyday inclusion for the benefit of their organizations' mission attainment and social impact. 


\section{REFERENCES}

Amir, Y.: 1969, 'Contact Hypothesis in Ethnic Relations', Psychological Bulletin, 71: 319-342.

Bradshaw, P., and C. Fredette; 2012, 'Determinants of the Range of Ethnocultural Diversity on Nonprofit Boards: A Study of Large Canadian Nonprofit Organizations', Nonprofit and Voluntary Sector Quarterly, 22(4): 391-409.

BoardSource: 2010, BoardSource Nonprofit Governance Index 2010 (Washington, DC, BoardSource).

BoardSource: (in review), Updated Report of the BoardSource Nonprofit Governance Index 2010, (BoardSource, Washington, DC).

Chavez, C., and J. Y. Weisinger: 2008, 'Beyond diversity Training: A Social Infusion for Cultural Inclusion', Human Resource Management, 47(2): 331-350.

Chin, W. W.: 1998, 'The Partial Least Squares Approach to Structural Equation Modeling', in G. A. Marcoulides (ed.), Modern Methods for Business Research (Lawrence Erlbaum Associates): 295.

Chin, W. W., and T. Frye: 1998, PLSGraph, Version 2.91.03.04.

Cox, P. L.: 2001, 'Teaching Business Students about Diversity: An Experimental Multimedia Approach', Journal of Behavioral and Applied Management, 2(2): 169.

Cox, T. H., S. A. Lobel, and P. L. McLeod: 1991, 'Effects of Ethnic Group Cultural Differences on Cooperative and Competitive Behavior on a Group Task', Academy of Management Journal, 34: 827-847.

Ely, R. J.: 2004, 'A Field Study of Group Diversity, Participation in Diversity Education Programs, and Performance', Journal of Organizational Behavior, 25: 755-780. 
Ely, R. J., and D. A. Thomas: 2001, 'Cultural Diversity at Work: The Effects of Diversity Perspectives on Work Group Process and Outcomes', Administrative Science Quarterly, 46: $229-273$.

Ely, R. J., I. Padavic, and D. A. Thomas: 2012, 'Racial Diversity, Racial Asymmetrie, and Team Learning Environment: Effects on Performance', Organizational Studies, 33(3): 341362.

Erhardt, N. L., J. D. Werbel, and C. B. Sharder: 2003, 'Board of Director Diversity and Firm Financial Performance', Corporate Governance, 11: 102-11.

Gefen, D., D. Straub, and M-C. Broudeau: 2000, 'Structural Equation Modeling and Regression: Guidelines for Research Practice', Communications of the Association for Information Systems, 4: Article 7.

Hanappi-Egger, E: 2012. "'Shall I stay or shall I go"? On the role of diversity management for women's retention in SET professions', Equality, Diversity and Inclusion: An International Journal, 31(2): 144-157.

Hair, J. F., W. C. Black, B. J. Babin, and R. E. Anderson: 2009, Multivariate Data Analysis, $7^{\text {th }}$ edition (Prentice Hall, Englewood Cliffs, NJ).

Hemphill, H., and R. Haines: 1997, Discrimination, Harassment and the Failure of Diversity Training: What to do Now (Westport CT, Greenwood Publishing Group).

Janssens, M., and P. Zazoni: 2007, 'What Makes an Organization Inclusive? Work Context and Diversity Management Practices Favoring Ethnic Minorities’ Inclusion', Paper presented at the Academy of Management conference, Philadelphia, PA. 
Liang, H., N. Saraf, Q. Hu, and Y. Xue: 2007, 'Assimilation of Enterprise Systems: The Effect of Institutional Pressures and the Mediating Role of Top Management', MIS Quarterly, 31(1): 59-87.

Miller T., and M. Triana: 2009, 'Demographic Diversity in the Boardroom: Mediators of the Board Diversity-Firm Performance Relationship', Journal of Management Studies, 46(5): 755-786.

Mor Barak, M. E.: 2000, 'Beyond Affirmative Action: Toward a Model of Diversity and Organizational Inclusion', Administration in Social Work, 23: 47-68.

Pavlou, P., H. Laing, and Y. Hue: 2007, 'Understanding and Mitigating Uncertainty in On-Line Exchange Relationships: A Principle-Agent Perspective', MIS Quarterly, 31(1): 105-136.

Pelled, L. H., G. E. Ledford, and S. A. Mohrman: 1999, 'Demographic Disparity and Workplace Inclusion', Journal of Management Studies, 36: 1013-1031.

Perry, L.B., and L. Southwell: 2011, 'Developing Intercultural Understanding and Skills: Models and Approaches', Intercultural Education, 22(6): 453-466.

Pettigrew, T.: 1998, 'Intergroup Contact Theory', Annual Review of Psychology, 49: 65-85.

Podsakoff, P. M., S. B. MacKenzie, J. Y. Lee, and N. P. Podsakoff: 2003, 'Common Method Biases in Behavioral Research: A Critical Review of the Literature and Recommended Remedies', Journal of Applied Psychology, 88(5): 879-903.

Podsakoff, P. M., and D. W. Organ: 1986, 'Self-Reports in Organizational Research: Problems and Prospects', Journal of Management, 12(4): 532-544.

Pollak, K. I., and Y. F. Niemann: 1998, 'Black and White Tokens in Academia: A Difference of Chronic Versus Acute Distinctiveness', Journal of Applied Social Psychology, 28(11): 954-972. 
Roberson, Q. M.: 2006, 'Disentangling the Meanings of Diversity and Inclusion in Organizations', Group \& Organization Management, 31: 212-236.

Shore, L. M., A. E. Randel, B. G. Chung, M. Dean, A. Dean, K. H. Ehrhart, and G. Singh: 2011, 'Inclusion and Diversity in Work Groups: A Review and Model for Future Research', Journal of Management, 37(4), 1262-1289.

Thomas, D., and R. Ely: 1996, 'Making Differences Matter: A New Paradigm For Managing Diversity', Harvard Business Review, 74(5): 79-90.

Walker, V. L., and D. J. Davidson: 2010, 'Vital Voices: Lessons Learned From Board Members of Color', Paper presented at ARNOVA's 39th Annual Conference, Alexandria, VA, November 18-20, 2010.

Weisinger, J. Y., and P. F. Salipante Jr.: 2007, ‘An Expanded Theory of Pluralistic Interactions in Voluntary Nonprofit Organizations', Nonprofit Management \& Leadership, 18(2): 157-173. 
Figure 1

\section{Conceptual Model}

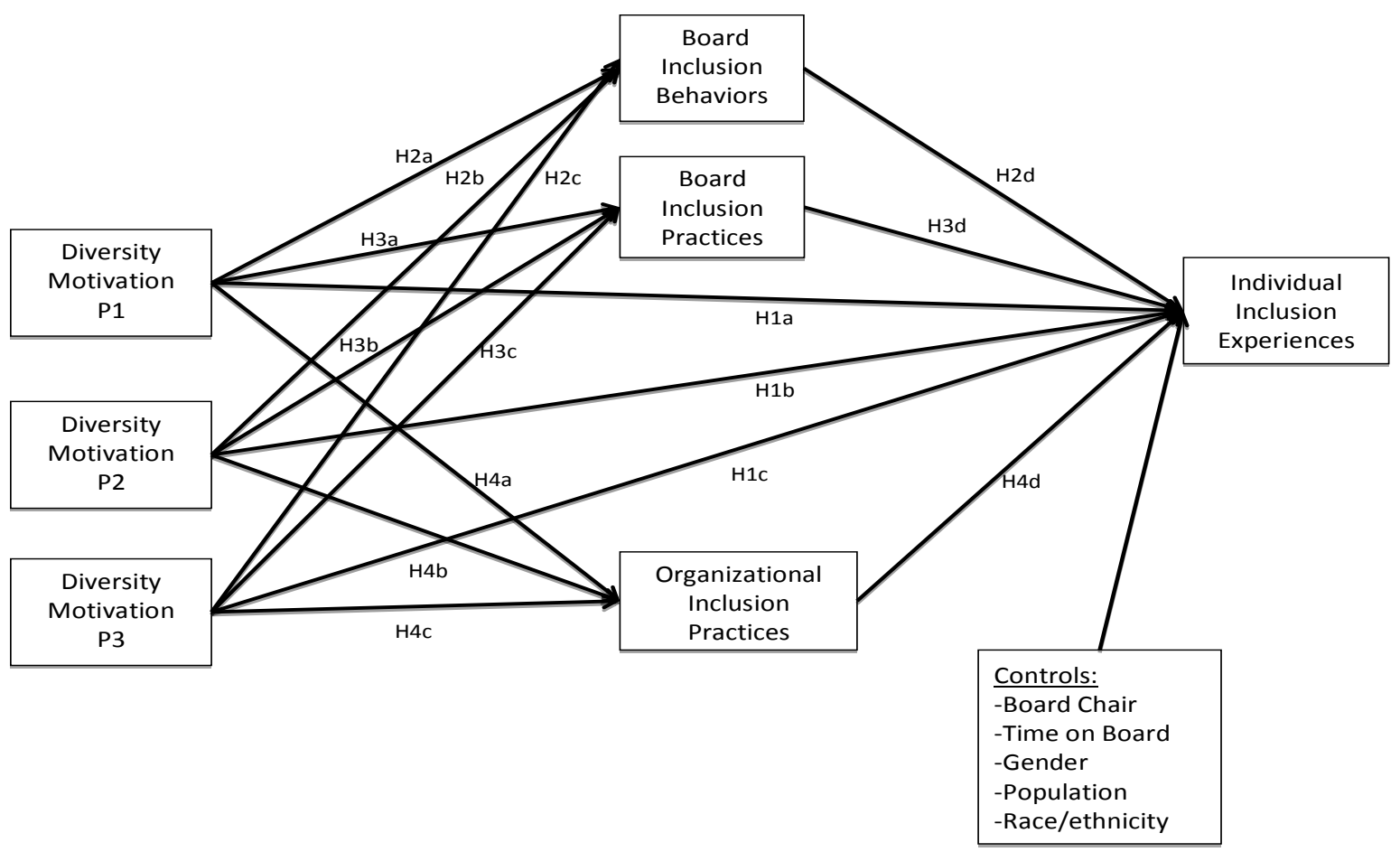


Figure 2A

Tests of Hypotheses for P1, P2 and P3 Diversity Perspectives: Operationalization 1

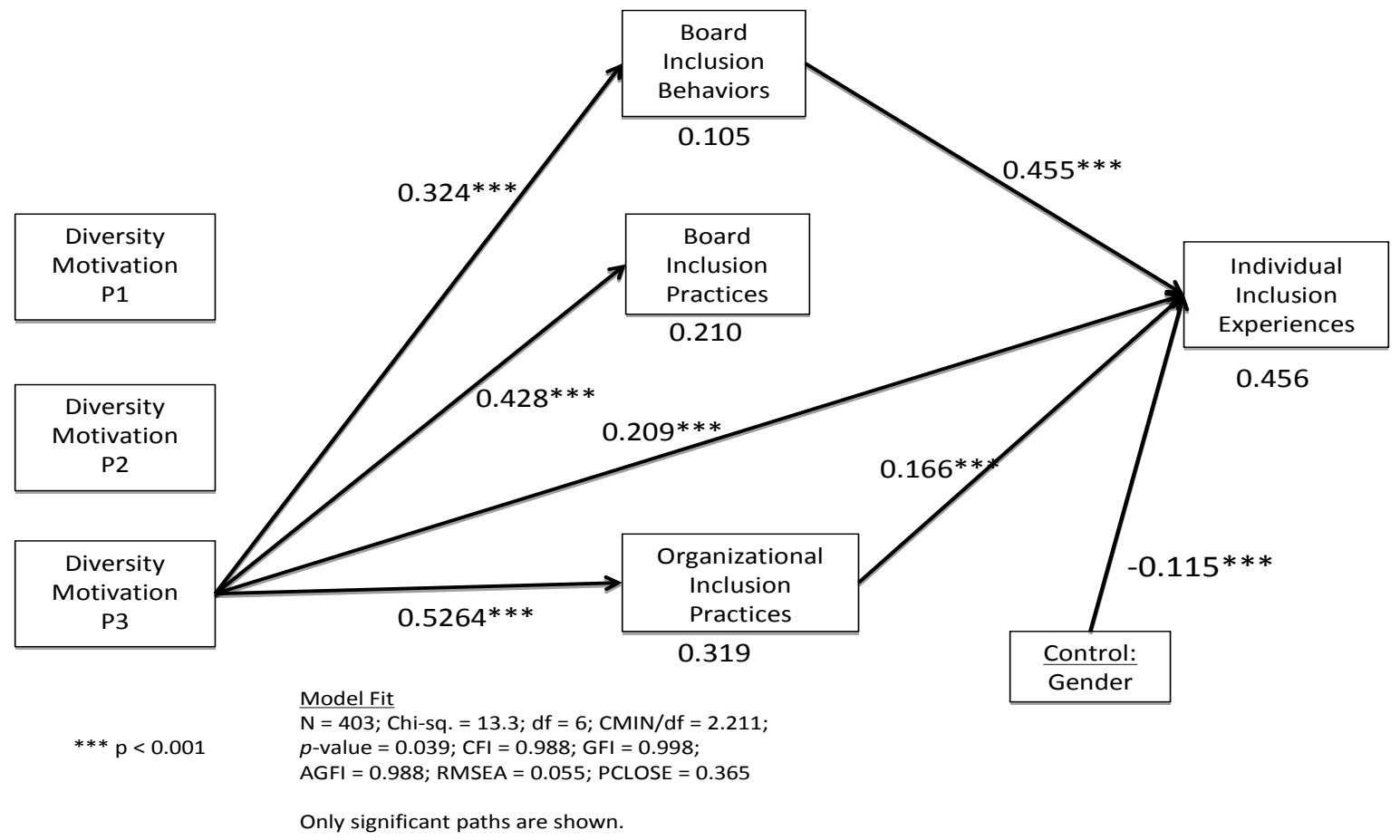




\section{Figure 2B}

\section{Tests of Hypotheses for P1E, P2E and P3E Diversity Perspectives: Operationalization 2}

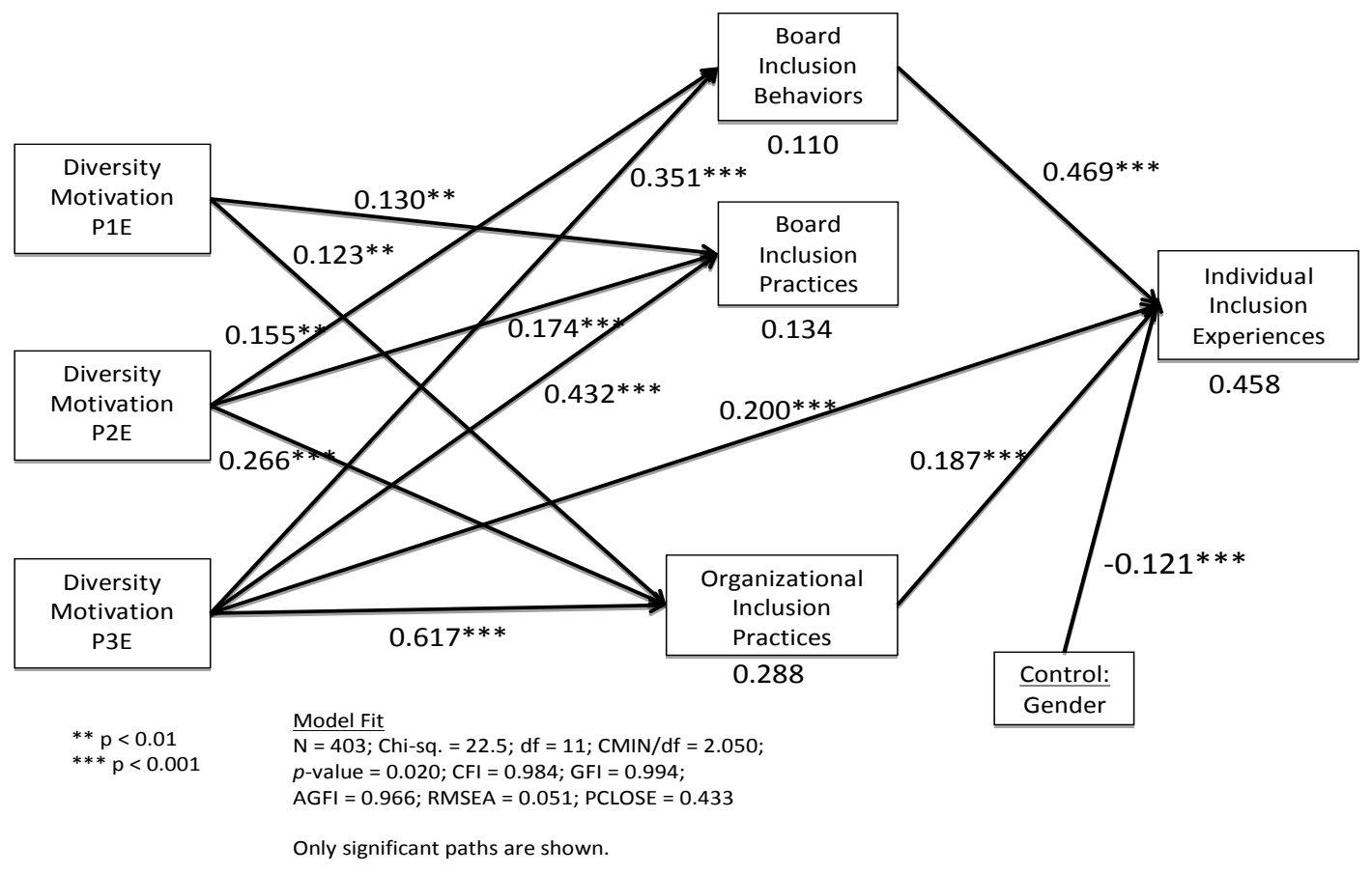




\section{Table 1}

\section{Demographics}

\begin{tabular}{|c|c|}
\hline Board Chair & Yes $-49.1 \%$; No $-48.1 \%$; No Response $-2.7 \%$ \\
\hline Length of Time on Boards & $\begin{array}{l}\text { Less than } 1 \text { year }-3.5 \% ; 1-3 \text { years }-11.2 \% ; 3-6 \text { years }-31.3 \% ; \text { more } \\
\text { than } 6 \text { years }-51.4 \% ; \text { no response } 2.7 \%\end{array}$ \\
\hline Gender & Female $-62.0 \% ;$ Male $-36.5 \%$; No Response $-1.5 \%$ \\
\hline Population & $\begin{array}{l}\text { Rural to semi-rural: }<50,000-7.9 \% \text {; Small urban area: } 50,000 \text { to } \\
249,000-12.4 \% \text {; Mid-size urban area: } 250,000 \text { to } 499,000-19.1 \% \text {; } \\
\text { Large urban area: } 500,000 \text { to } 1 \text { million }-23.3 \% \text {; Mega urban area: } \\
\text { greater than } 1 \text { million }-37.2 \% \text {. }\end{array}$ \\
\hline Race/Ethnicity & $\begin{array}{l}\text { African American/Black - 70.2\%; American Indian/Alaska Native - } \\
2.0 \% \text {; Asian - } 10.2 \% \text {; Hispanic/Latino/Spanish }-13.6 \% \text {; Native } \\
\text { Hawaiian/Pacific Islander }-0.7 \% \text {; Other }-1.0 \% \text {; Two or more races - } \\
2.0 \% \text {; No Response }-1.0 \%\end{array}$ \\
\hline
\end{tabular}

Table 2A

Descriptive Statistics and Correlations for P1-P3 (Operationalization 1 of Diversity Perspectives)

\begin{tabular}{|l|l|l|l|l|l|l|l|l|l|l|l|l|}
\hline & Mean & SD & CR & AVE & MSV & P1 & P2 & P3 & BIB & BIP & OIP & IIE \\
\hline $\begin{array}{l}\text { Discrimination-and- } \\
\text { Fairness (P1) }\end{array}$ & 2.65 & 0.903 & 1.000 & 1.000 & & $\mathbf{1 . 0 0 0}$ & & & & & \\
\hline $\begin{array}{l}\text { Access-and- } \\
\text { Legitimacy (P2) }\end{array}$ & 2.72 & 0.791 & 0.904 & 0.824 & 0.03 & 0.474 & $\mathbf{0 . 9 0 8}$ & & & & & \\
\hline $\begin{array}{l}\text { Integration-and- } \\
\text { Learning (P3) }\end{array}$ & 2.59 & 0.785 & 0.854 & 0.745 & 0.06 & 0.495 & 0.857 & $\mathbf{0 . 8 6 3}$ & & & & \\
\hline $\begin{array}{l}\text { Board Inclusion } \\
\text { Behaviors }\end{array}$ & 1.75 & 0.649 & 0.893 & 0.585 & 0.18 & 0.110 & 0.283 & 0.324 & $\mathbf{0 . 7 6 5}$ & & & \\
\hline $\begin{array}{l}\text { Board Inclusion } \\
\text { Practices }\end{array}$ & 2.06 & 0.826 & 0.910 & 0.716 & 0.08 & 0.281 & 0.392 & 0.428 & 0.000 & $\mathbf{0 . 8 4 6}$ & & \\
\hline $\begin{array}{l}\text { Organizational } \\
\text { Inclusion Practices }\end{array}$ & 2.10 & 0.667 & 0.915 & 0.607 & 0.15 & 0.326 & 0.503 & 0.564 & 0.256 & 0.542 & $\mathbf{0 . 7 8 0}$ & \\
\hline $\begin{array}{l}\text { Individual Inclusion } \\
\text { Experience }\end{array}$ & 3.20 & 0.701 & 0.894 & 0.679 & 0.10 & 0.289 & 0.407 & 0.461 & 0.581 & 0.198 & 0.541 & $\mathbf{0 . 8 2 4}$ \\
\hline
\end{tabular}

Notes.

$\mathrm{CR}=$ Composite reliability: confirmed with item loadings exceeding the minimum level (.50), or better (.70) (Chin, 1998). AVE = Average variance explained. Convergent validity is established when CR is greater than average AVE, and AVE is greater than 0.5 . Discriminant validity is established when maximum shared variance (MSV) is less than AVE and by demonstrating that the correlation between any two constructs is less than the square root of AVE (Gefen, Straub, \& Broudeau, 2000) which is highlighted along the diagonal. $\mathrm{N}=403$. 
Table 2B

Descriptive Statistics and Correlations for P1E-P3E (Operationalization 2 of Diversity Perspectives)

\begin{tabular}{|c|c|c|c|c|c|c|c|c|c|c|c|c|}
\hline & Mean & SD & $\mathrm{CR}$ & AVE & MSV & P1E & $\mathrm{P} 2 \mathrm{E}$ & P3E & BIB & BIP & OIP & IIE \\
\hline $\begin{array}{l}\text { Discrimination- } \\
\text { and-Fairness P1E) }\end{array}$ & 0.15 & 0.359 & 1.000 & 1.000 & & 1.000 & & & & & & \\
\hline $\begin{array}{l}\text { Access-and- } \\
\text { Legitimacy (P2E) }\end{array}$ & 0.13 & 0.338 & 1.000 & 1.000 & 0.03 & -0.164 & 1.000 & & & & & \\
\hline $\begin{array}{l}\text { Integration-and- } \\
\text { Learning (P3E) }\end{array}$ & 0.43 & 0.496 & 1.000 & 1.000 & 0.06 & -0.370 & -0.341 & 1.000 & & & & \\
\hline $\begin{array}{l}\text { Board Inclusion } \\
\text { Behaviors }\end{array}$ & 1.75 & 0.649 & 0.893 & 0.585 & 0.18 & -0.161 & 0.035 & 0.298 & 0.765 & & & \\
\hline $\begin{array}{l}\text { Board Inclusion } \\
\text { Practices }\end{array}$ & 2.06 & 0.826 & 0.908 & 0.713 & 0.08 & -0.058 & 0.005 & 0.325 & 0.582 & 0.844 & & \\
\hline $\begin{array}{l}\text { Org Inclusion } \\
\text { Practices }\end{array}$ & 2.10 & 0.667 & 0.915 & 0.607 & 0.15 & -0.149 & 0.036 & 0.481 & 0.203 & 0.001 & 0.779 & \\
\hline $\begin{array}{l}\text { Individual } \\
\text { Inclusion } \\
\text { Experience }\end{array}$ & 3.20 & 0.701 & 0.894 & 0.678 & 0.10 & -0.126 & -0.007 & 0.432 & 0.402 & 0.259 & 0.543 & 0.823 \\
\hline
\end{tabular}

Notes.

CR = Composite reliability: confirmed with item loadings exceeding the minimum level (.50), or better (.70) (Chin, 1998). AVE = Average variance explained. Convergent validity is established when CR is greater than average AVE, and AVE is greater than 0.5 . Discriminant validity is established when maximum shared variance (MSV) is less than AVE and by demonstrating that the correlation between any two constructs is less than the square root of AVE (Gefen, Straub, \& Broudeau, 2000) which is highlighted along the diagonal. $\mathrm{N}=403$. 
TABLE 3

Summary of Results of Tests of Hypotheses

\begin{tabular}{|l|l|l|l|}
\hline & Description* & $\begin{array}{l}\text { Operationalization 1: } \\
\text { P1, P2, P3 }\end{array}$ & $\begin{array}{l}\text { Operationalization 2: } \\
\text { P1E, P2E, P3E }\end{array}$ \\
\hline H1a & Weak association between P1 and IIE. & No Association & No Association \\
\hline H1b & Moderate association between P2 and IIE. & No Association & No Association \\
\hline H1c & Strong association between P3 and IIE. & Supported & Supported \\
\hline H2a & Weak association between P1 and BIB. & No Association & No Association \\
\hline H2b & Moderate association between P2 and BIB. & No Association & Supported \\
\hline H2c & Strong association between P3 and BIB. & Supported & Supported \\
\hline H2d & BIB positively associated with IIE. & Supported & Supported \\
\hline H3a & Weak association between P1 and BIP. & No Association & Supported \\
\hline H3b & Moderate association between P2 and BIP. & No Association & Supported \\
\hline H3c & Strong association between P3 and BIP. & Supported & Supported \\
\hline H3d & BIP positively associated with IIE. & No Association & No Association \\
\hline H4a & Weak association between P1 and OIP. & No Association & Supported \\
\hline H4b & Moderate association between P2 and OIP. & Supported & Supported \\
\hline H4c & Strong association between P3 and OIP. & Supported & Supported \\
\hline H4d & OIP positively associated with IIE. & Supported & Supported \\
\hline N & & &
\end{tabular}

Notes.

* $\mathrm{P} 1$ = Discrimination-and-Fairness perspective, $\mathrm{P} 2=$ Access-and-Legitimacy perspective, and $\mathrm{P} 3=$ Integration-and-Learning perspective. $\mathrm{P} 1 \mathrm{E}$ $=$ Discrimination-and-Fairness perspective above the median, $\mathrm{P} 2 \mathrm{E}=$ Access-and-Legitimacy perspective above the median and encompassing the effect of $\mathrm{P} 1$, and $\mathrm{P} 3 \mathrm{E}=$ Integration-and-Learning perspective above the median and encompassing the effects of P1 and P2. IIE $=$ Individual Inclusion Experiences, $\mathrm{BIB}=$ Board Inclusion Behaviors, BIP $=$ Board Inclusion Practices, and OIP = Organizational Inclusion Practices. 